\title{
Improving the capabilities of project team management using the \\ Gestalt Cycle of Experience
}

Zwikael, O., Bar-Yoseph, B. (2004). Improving the capabilities of project team management using the Gestalt cycle of experience. Team Performance Management, 10 (7), 137-144.

\begin{abstract}
Tools that project managers use while managing project teams are insufficient. This probably contributing to the large number of projects that fail to achieve their objectives within the desired time and budget to the full satisfication of the customer. An inovative approach for the identification of the main reasons for these failures and the ways to prevent them are described in this paper. The paper introduces several solutions for project team building and management, based on an interdisciplinary approach and theory known in the Gestalt Cycle of Experience. In spite of their differences, the humanistic process of need fulfillment and the project management approach have a lot in common. The present paper demonstrates that both team decision making and project management methods use similar processes. Hence, applying the well known mental barriers and solutions from the Gestalt Cycle of Experience may reduce the currently high percentage of project failures. The paper finally introduces several areas in which project managers can focus on when dealing with managing a project team.
\end{abstract}




\section{Background}

A project is a temporary endavor undertaken to create a unique product or service (PMI Standards Committee, 2000). However, most of the projects do not successfully achieve that aim according to the desired specifications and within the specified budget and the promised time schedule, while keeping the customer and stakeholders happy (Johnson et. al, 2001). These disappointed results call for the possibility that there may still be problems in managing a project that have not been properly considered yet.

The traditional literature of project management deals mostly with the technical aspects, while too little attention is being paid to team building processes. For example, the Project Management Body of Knowledge (PMI Standards Committee, 2000), known as the PMBOK, examines the human resource aspects as one of its nine knowledge areas. The processes that are included in this knowledge area are "organizational planning", "staff acquisition" and "team development". Tools that are suggested within these processes include training, reward systems, recognition systems and team building activities. These tools do not provide the project manager with the necessary skills for dealing with interruptions, which may rise during the project.

Therefore, additional approach for project team management is needed to improve project sucssess. For this purpose, this paper uses a well known approach from the psychological dicipline. This natural process approach, called Gestalt Cycle of Experience (Clarkson, 1989; Nevis, 1987; Wheeler, 1991; Haris, 1989; Houston, 1998 and others), is implemented in this paper in order to suggest a different focus to a project manager while managing project teams. In this paper, we first introduce the Gestalt Cycle of Experience (COE). Then, we compare its phases with those of the project management process. After identifying the similarity between the individual need fulfillment process and the 
organizational project management process, we offer a project manager areas to focus on during the process of decision making.

\section{Introduction}

The Gestalt COE was developed at the turn of the $20^{\text {th }}$ century in the Cleveland Gestalt Institute. It models the psychologic process of need fulfillment by an organism or a group. The Gestalt COE demonstrates how interest shifts from one subject to another through the process.

Whether described as a continuous wave or as an infinite circle, the Gestalt COE, or in its original name "The need fulfillment process", is one of the ways in which the humanist describes the process of fulfilling a need (Nevis, 1996). The need can be a simple urge for food caused by a sense of hunger, which leads to an action of looking for food and eating until hunger is no longer felt. A healthy process is a self regulating one, which is done without interference of conscience or emotions, and finaly leads to equilibrium. An unhealthy process will be unsmooth and the people involved will display nervousness. The model shows that there are seven phases in this process, each having its own unique characteristics.

This theory is based on the belief that it is impossible to explain complex human behavior as an additive structure of simple items. According to Gestalt psychology, experience is an aggregation of basic behavioral "atoms". For example, in Exhibit 1 the reader will see a square. Only later, he will "dismantle" it to four bars connected at $90^{\circ}$ angels. Gestalt psychology shows that people react to the inner pattern of things, not to parts of this pattern. Experiments done with blind people, who gained sight, showed that pattern perception is a basic human quality even for people who cannot name the patterns. Moreover, this is not a passive reaction, since people force order on what they see. 


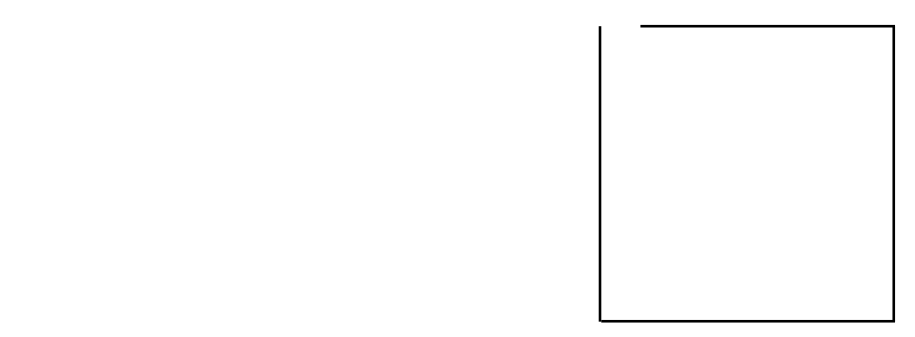

Exhibit 1 - Four Lines Connected to Form a 90-degree Angle (or a square?)

The COE summarizes the process in which a human being, as an individual or part of a collective, becomes aware of what is going on at any given moment with himself or in his environment. The human being then mobilizes this energy to perform some action, which deals with the possibilities offered by this new awareness. The process assumes that when a person becomes aware of a state of imbalance, his natural desire is to act in order to reach a new state of equalibrium. The model also assumes that there is a natural tendency for the (normal) human being to perform in the most efficient and effective way. Understanding this process is the key to effective performance while managing people.

The Gestalt COE integrates the behavioral aspects to one unit of human experience. The ability of individuals (or systems) to move uninterruptedly through the phases in the COE is necessary for healthy learning and functioning. When an individual or a group develops awareness for a need at a given moment and mobilizes the energy to perform the necessary action, we can talk about a healthy human process. 
In order to understand the $\mathrm{COE}$ approach, we first introduce two principles, which are the baseline for this paper. The "Principle of figure formation" is the result of paying attention (awareness), for example, being aware of a certain specific tree while walking in a forest. When we pay attention to the specific tree, it looks more clearly and is separated from the general background. The "Pragnanz principle" is the law of equilibrium. This principle claims that any experience tends to be "useful" in as much as the sensory terms enable it to be. This means that like the same principle in physics, psychological behavior does not go in an arbitrary direction, but rather in the direction that will bring back the equilibrium and the "useful" pattern. This principle says that people tend to actively finish activities (reach a state of equilibrium) and will not rest untill they do so. The phases in which they finally reach equilibrium will be introduced next.

\section{The Gestalt Cycle of Experience Phases}

The Gestalt $\mathrm{COE}$ is set to solve a problem to an individual or a group from sensing the problem until the achievement of equilibrium again. The COE contains seven phases, as can be seen in Exhibit 2 (Nevis, 1987).

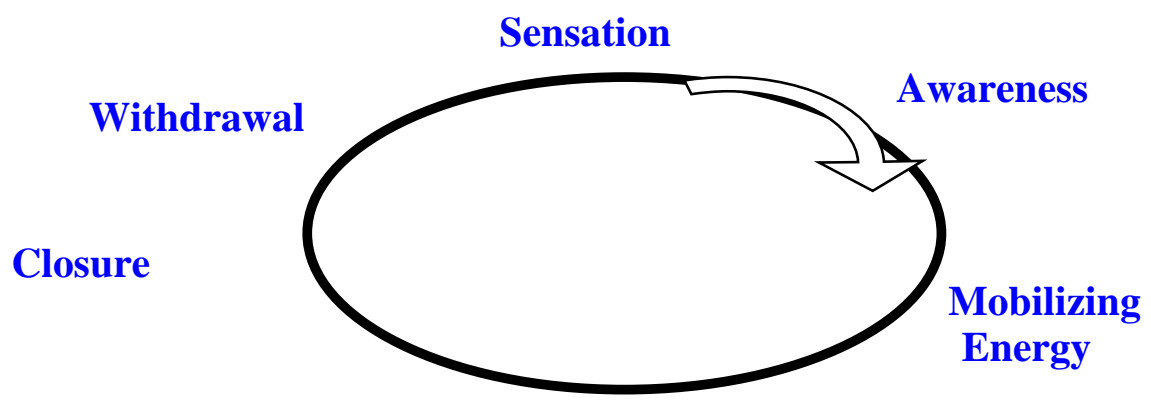

Contact

Action

\section{Exhibit 2 - Gestalt Cycle of Experience (COE) Phases}


The process begins with the sensation phase, with which every organism or team begins any change from a state of equilibrium. In this phase, we use our senses to collect information and identify disruption of this equilibrium, i.e. unsatisfied customer. The sensation phase leads to the phase of awareness, in which the organism is aware of a certain need, i.e. improving the support that the customer gets from the organization. The phases of sensation and awareness are the sensory phases, where one gathers information about the environment and become aware of some part of this information.

The awareness phase leads to the mobilizing energy phase, when the team mobilizes its energy towards action. All forces concentrate in order to analyze the problem from all aspects and gather the necessary resources. For example, deciding about the best alternative that can solve the problem of the customer.

The action phase is the first operational one. In this phase, the team ties together the aroused energy, behavioral skills and knowledge in a way that brings forth an appropriate action, in order to fulfill its need, for example add new members to the support team.

The next phase is the contact phase, in which the team makes contact with the problem in order to attain need satisfaction. Satisfaction refers to the experience as to how, where, and in what way is the need met. In the previous example, the customer may find that the solution was not satisfied, so more employees may be hired. Contact is performed by breaking the boundary between the team and the environment. The question "is the action fulfilling the need?" is asked at the (virtual) boundary of the contact with the problem. The boundaries of the problem (the need) are destroyed, the need is fulfilled and the team advances to the next stage.

The closure is the phase in which the solution is accepted and the pattern reaches closure (meaning, it returns to a state of equilibrium), until a withdrawal is noticed and the cycle begins again. 


\section{The Project Management Phases}

Both the project and the Gestalt COE are temporary processes aimed at solving a problem that a team or an organization is facing. Both have a starting point and upon its completion all parties involved are ready for the next project. Literature of project management describes the phases of the project in several ways. Lokyer \& Gordon (1996) perceive a project as a process with four phases:

\section{Conception $\rightarrow$ Development $\rightarrow$ Realization $\rightarrow$ Termination}

Maylor (1999) sees project management as a process of three general phases which he calls:

\section{Design it $\rightarrow$ Do it $\rightarrow$ Develop it}

Stevenson (2002) recognizes four phases:

\section{Definition $\rightarrow$ Planning $\rightarrow$ Execution $\rightarrow$ Delivery}

However, PMBOK (PMI Standards Committee, 2000) is the most recognized body of knowledge and is also recognized as a standard by the American National Standard Institute (ANSI). According to the PMBOK a project has four phases, including:

\section{Initiation $\rightarrow$ Planning $\rightarrow$ Execution $\rightarrow$ Closing}

Initiation is the phase of formally authorizing a new project. This phase links the project to the ongoing work of the performing organization. Projects are typically authorized as a result of one or more of the following: A market demand, a business need, a customer request, a technology advance or a social need.

Planning processes define and refine objectives and select the best of the alternative courses of action to attain the objectives that the project was undertaken to address. Planning is of major importance to a project because the project involves doing something that has not been done before. 
Executing processes coordinate people and other resources to carry out the plan in order to perform the project. Finally, closing processes formalize acceptance of the project and bring it to an orderly end.

\section{Comparing the Phases of the Gestalt COE and a Project}

In order to implement the ideas shown in the $\mathrm{COE}$, we first have to prove the existance of a similarity between the environment and phases of both the Gestalt COE and project management. While the Gestalt COE was developed for the personal or team level system, project management describes a process, which is at a higher level system. The adaptation of the therapeutic model to a higher system level was already demonstraited (i.e. Wheeler, 1991; Bar-Yosef, 2001). If we examine the operating fields of the project management and the Gestalt view, we can compare the Organism/Environment $[\mathrm{O} / \mathrm{E}]$ system in Gestalt to a Project/Environment [P/E] system in Project Management.

The field of activity of the Gestalt is comprised of the organism and the environnment. For example, the hungry individual and people surronding him. In the same way, the field of activity of the project manager is comprised of the Project and the Environment. The project manager, much like the organism, has to consider the environment so as to satisfy needs.

In order to relate to the P/E system, the project manager should understand the system as a whole and the role of each part of the system in the context of the whole. Indeed, for a successful project, the project manager may think of the project, its environment and its boundaries, as illustrated in Exhibit 3. While the project may include the project manager and the project team, the environment of the project includes all stakeholders - customers, sub contractors, functional managers in the organization, etc. 


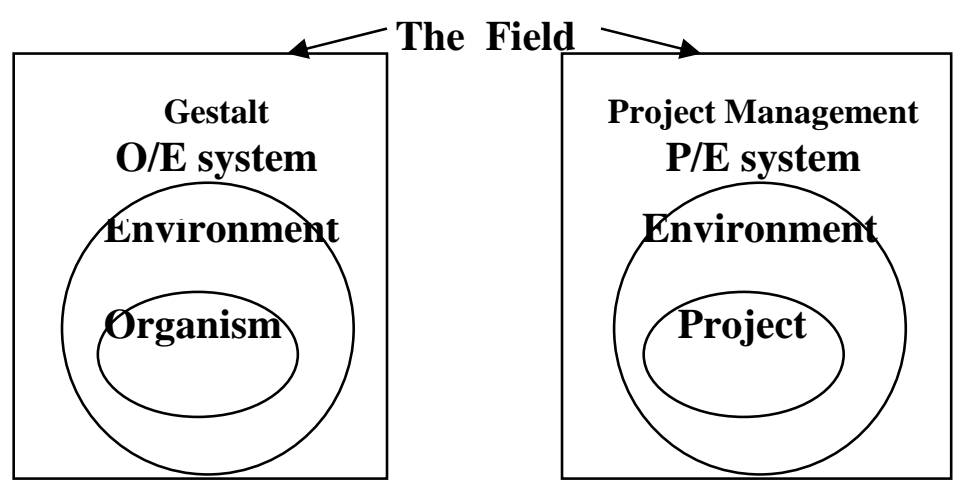

Exhibit 3 - Organism and Project Operating Areas

Once identifying the similarity between the environment of both the Gestalt COE and project management, the phases can be also compared. The sensation and awareness phases of COE are actually defining the needs that cause the imbalance and suit the pre-project phase, in which the environment is scanned for a possible problem to be solved. Hence, these first two Gestalt phases fit into the project initiation phase. In project management, market research is a possible process performed during this phase. If the market is unbalanced (out of equilibrium), it leads to the phase of awareness. Well defined needs are a precondition for any successful project and should lead to a mobilization of the energy towards finding a solution.

The mobilizing energy phase, which can be compared to the project management planning phase, may involve many disciplines and requires team effort. Team work is needed to plan the solution and the processes that are required to be performed.

In the world of project management, once the plan is completed, the execution phase can begin. Execution creates the desired contact between the environment and the project. Similarly, in COE, action and contact will happen in the boundary between the environment and the individual. 
The process continues with the closing phase. The organism has resolved the need and is closing the process, while the project manager concludes the project in the closure phase, makes conclusions and completes the project documentation.

After the withdrawal phase, the organism is in equilibrium and is ready for the next cycle. Similarly, once the project is completed, a situation of withdrawal is reached. This phase will prevail until a new state of imbalance is sensed and awareness is raised to a new need, in which case the whole cycle will begin again. The comparision between the Gestalt Cycle of Experience and the project management phases is illustrated in Exhibit 4.

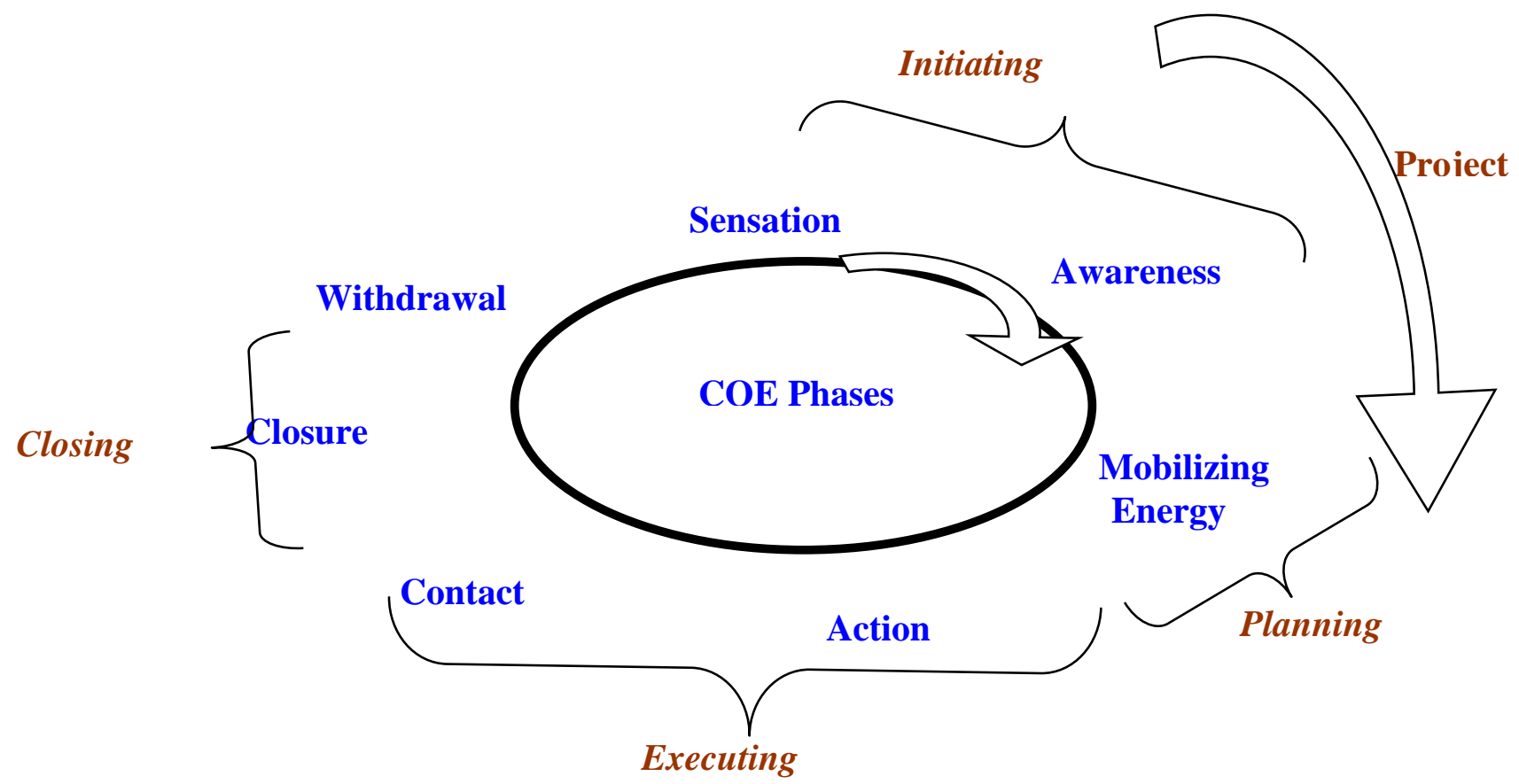

Exhibit 4 -Comparing the Gestalt Cycle of Experience with the Project Management Phases 
As can be seen in Exhibit 4, project management processes are being performed in the way that the Gestalt theory identifies the need fulfillment process. Not only that similar phases were found, but both models have similar objectives, which may be finding and implementing the best solution to a specific problem. That similarity can elevate project manager's capabilities if we identify known interruptions from the need fulfillment process and implement the appropriate solutions in the project management environment.

\section{Interruptions in the Gestalt COE Process}

Failing to complete the cycle of experience would obviously leave the individual or team dissatisfied. In the same way, an interrupted project cycle will result in an incomplete or sub-optimal execution of the project. Any distraction from the "normal" path of the process should yield sub optimal results. Hence, one has to "close the Gestalt", meaning to complete the cycle through all its phases, while a project manager insists on going through all the phases of the project. Unfortunately, along the Cycle of Experience and the project process, interruptions rise and destroy the smooth running of the process.

If we accept the analysis described above of the similarity between the Gestalt COE and the project life cycle, we have to agree that each phase of the project carries with it a different state of mind if not a different culture. For example, the sensation phase of the COE calls for a lot of sensitivity and imagination, while the energy mobilizing phase calls for less imagining and more action. The shift between phases calls, therefore, for a change of a state of mind. It is only natural to expect interruptions in switching from a certain state of mind to another. Since these interruptions are of mental rather than of technical nature, a humanistic approach may be used for identifying, dealing and hopefully eliminating the interruptions. Clearly, a good project team with a lot of experience in working together can overcome interruptions. 
Interruptions interfere with the smooth transfer from phase to phase. Nevis (1987) states that the system should be aware of the forces acting for and against while moving to a new position. Interruptions were identified and solutions were found in the Gestalt theory. Where there is a driving force for change, a complementary force exists against it. Interruption is not an absence of energy, but rather energy in a different direction (Rainy, 1993). Interferences or disturbances at any part of this cycle naturally interfere with the elegant and natural rhythm of the need fulfillment process (Clarkson, 1989).

The six most important interruptions (Clarkson, 1989) introduced in COE will now be demonstrated. Each interruption characterizes one COE phase, as is demonstrated in Exhibit 5. The connection of interruption type to a certain specific phase of the COE is typical, but by no means absolute.

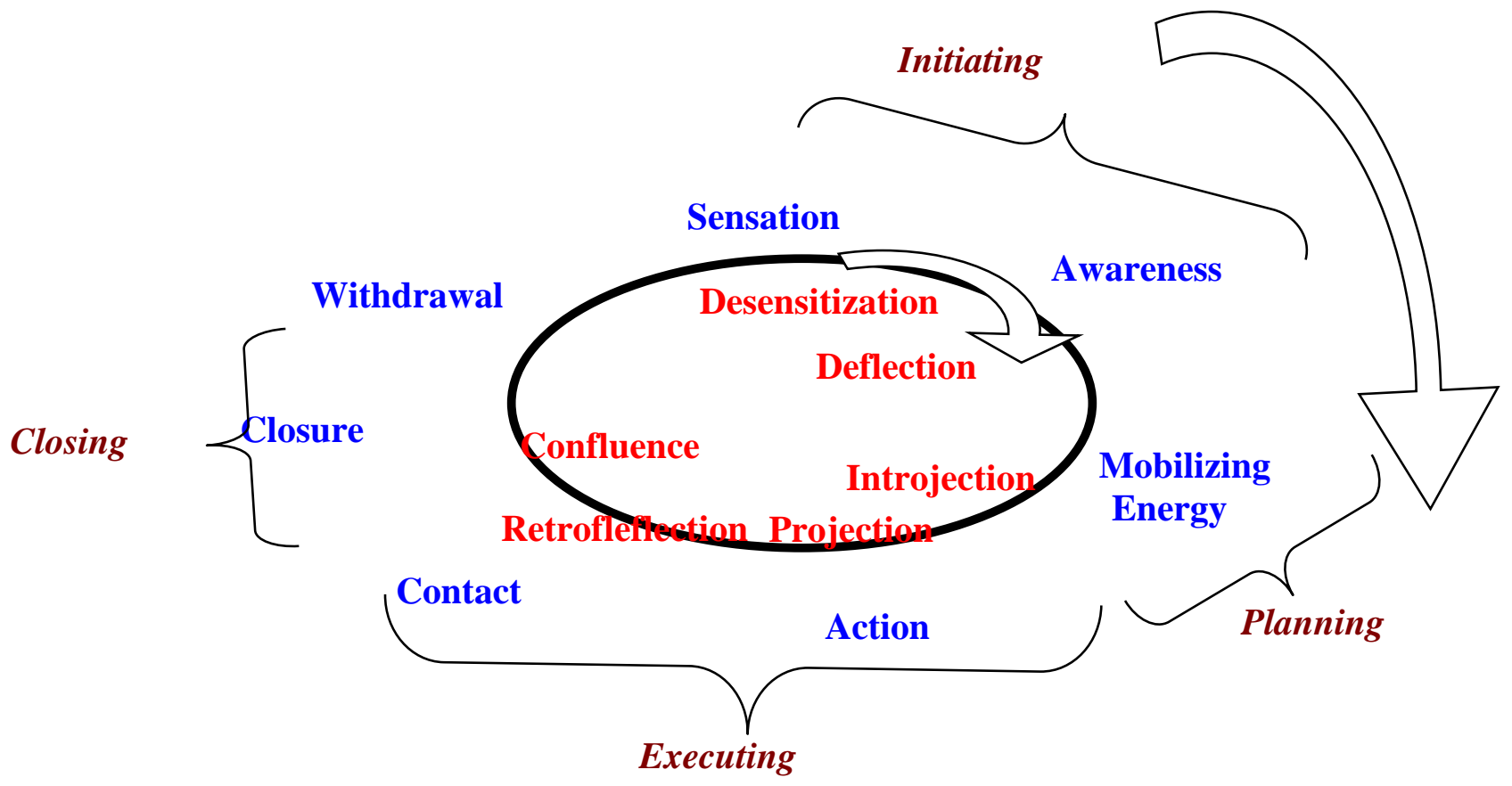

Exhibit 5 - The Gestalt COE, Project Management Phases and interruptions 
1. The interruption that is most likely to appear in the first phase of the Gestalt COE (sensation) is Desentization. This is a gap in sensory ground, lessened permeability of the boundaries with the environment or, in simple words, being less sensitive to inner and environmental stimulants. For example, the team does not understand the disappointment of the customer from the service, due to lack of signals or misinterpretation.

2. The interruption of Deflection appears usually in the awareness phase. It is characterized by behaviors of avoidance and is defined as "Turning aside from direct contact, making it vague, generalized or bland and avoiding the impact of the stimuli from self or the environment". In the example, the team will fail to interpret a feeling of disappointment from service by the customer (assuming it is due to functionality problem of the product, for example).

3. The interruption of Introjection is likely to appear in the mobilizing energy phase. This is a mechanism of swallowing and accepting without digesting. By doing this, one may find himself with two opposing mandates. In the example, asking the product manager to immediate improve the functionality of the product, while service is actually the core problem.

4. The action phase raises the interruption of Projection, which results in holding on to own beliefs. This is, actually, the other side of the coin of introjection. The person will prescribe to others what he discovers in himself. In the example, the team will apologize of insufficient product performance to every new customer.

5. The final contact phase raises the interruption of Retroflection, turning energy, which is intended for external use to oneself. In the example, serving other customers may be damaged, since energy is dedicated to product improvement. 
6. The resolution and closure phase raises the interruption called Confluence, which is defined as "pedaling in the same direction". This interruption is characterized by being agreeable and minimizes differences. Total lack of criticism may lead to close without real and full resolution of the problem. In the example, the team may close the process without solving the customer's problem, since others still show signs of satisfaction.

\section{Interruptions in the Project Management Process}

Recognizing the similarity between the Gestalt $\mathrm{COE}$ and project management processes, one may assume that similar interruptions may be recognized in both processes. The Gestalt COE interruptions will be implemented and demonstrated on a project management case.

In this case, Operations VP tries to solve a problem that troubles all three departments under his responsibility - Production, Inventory and Procurement. Customers, including Marketing and Engineering divisions, have complained that information received from the Operations division is incorrect and incomplete.

It will be naive to claim that it is possible to write a handbook with interruptions and solutions. Even though we can point out interruptions and typical solutions, the solutions don't work like magic. In some cases, the solution consists of one action performed by the project manager, while in others the solution is a process that may take quite a while. Hence, we may suggest some areas of focus to the project team, based on COE solutions to known interruptions. 


\section{Interruption 1 - Desensitization}

Desensitization was introduced as an interruption that is caused while the team is insensitive to inner and environmental stimulants. This interruption is connected to the sensation phase of the $\mathrm{COE}$ and therefore may rise during the Initiation phase of the project. For example, although Marketing VP complains about schedule overruns and incorrect information, the Operations VP ignores the problem. The gathering of information isn't appropriate, the problem is not identified and the project doesn't begin.

The solution to this interruption, as suggested in the COE, will be to increase the amount of sensing and to develop awareness to small chunks of information. Asking the customers for their opinion may do it. Being sensitive and aware of pieces of the whole problem may help the Operations VP to identify some of the problems taking place in his own territory. For example, a meeting with each department manager may show that the Inventory manager cannot report accurate data about the inventory in the warehouse; The Procurement manager faces problems with the quantities of each order and the Production manager has trouble making the marketing deadlines.

\section{Interruption 2 - Deflection}

This interruption is likely to appear in the initiation phase of the project, where there should be an understanding that 'we face a problem', but the problems cannot be stated very clearly. For example, the Operations VP avoids acknowledgment of departmental problems. He does not understand, or refuses to admit, that there is an information problem in each department that is caused by a general and larger problem. Delegating some of the authority to people from different departments and/or consulting with an external expert can solve this problem. 


\section{Interruption 3 - Introjection}

Introjection usually appears in the mobilization phase of the COE, and therefore may be expected to rise in the planning phase of a project. It will result in a total lack of criticism to information and ideas gathered during the previous phase. It is easy to understand that this interruption is the result of staying in a state of mind that fits the previous phase.

In order to overcome this interruption, one should scrutinize assumptions and beliefs and explore the consequences. To do so, the project manager should take each problem apart and solve it. This process may show Operations VP that using software for control may solve the inventory problem. The same software can indicate the procurement manager how many items should be purchased, reduce the amount of shortage and help the production manager make his deadlines.

\section{$\underline{\text { Interruption } 4 \text { - Projection }}$}

This interruption may rise during the action phase of the project, after the Inventory manager was chosen to be the project manager. This may cause an identification of internal problems as external ones and using internal resources when external ones are needed. In this case, the project manager may see the inventory problems alone, while not being able to see the big picture. The new software may then be focused on the inventory problems, with no solutions to problems of other departments. Increasing individual responsibility and delegation should contribute to solve this interruption, for example building a project team that includes representatives from other departments as well. Those members will have the power to change things only if they work together with full authority and cooperation. An integrated solution may be found, and an Enterprise Resource Planning (ERP) solution can be addressed as the best solution to that problem. 


\section{$\underline{\text { Interruption } 5 \text { - Retroflection }}$}

This interruption may rise during the phase of contact of COE or the execution phase of the project. The contact may take place only on the boundary of the project manager's own problems, leaving other problems not completely solved. For example, when the project manager faces budget constraints or cuts during the project, he may decide to implement only inventory modules of the ERP software, while the implementation of other modules are delayed in few months. That may not be very helpful in solving the main problem of the division. The solution suggested in the Gestalt theory is adhering to the original plan, encouraging more contact or providing a base for small contact steps along the entire plan, meaning trying to insist on solving the identified problems, despite of occurring changes.

\section{Interruption 6 - Confluence}

During project closure, the project manager accepts any criticism made by team member and customers. This may cause the project never to reach completion. This interruption, although arises at the end of the project, must be dealt with at the planning phase. A clear project scope, clear boundaries between customers and team members and developing awareness to differences and increasing conflict-handling skills should help overcome this interruption. Criticism should be checked out very seriously, by a comparison of project deliverables to the project objectives and specification documents. A firm and rigid checklist may help the project manager in completing this task. 
It is interesting to note that solving most interruptions involves teamwork. This is easy to accept if we note that most interruptions dealt with in this paper are mental and have their roots in the character of the manager. Management by a team can ensure balanced tendencies and solve interruptions. The practical solution will be the participation of all stakeholders during all phases of the project, may it be customers, relevant resources and functional managers, sub-contractors or the management of the project manager himself. The forum can be a steering committee, professional committee or project reviews.

\section{Summary}

This paper showed the similarity between two approaches from different disciplines. Both project management and Gestalt Cycle of Experience follow the same basic routine and phases. It was demonstrated how project phases follow the Gestalt Cycle of Experience to get from a broken equilibrium in a system back to the withdrawal phase of the same system in a modified form. Both approaches are perceived as basic routines for problem solving and decision-making.

In this paper, Gestalt solutions were used to improve the capabilities of project team management. Some conclusions that can help a project manager may be:

1. Each phase of the project gives rise to a different type of interruption. The interruptions are rooted in the difficulty in switching from the present state of mind to the state of mind needed for the next phase.

2. The project team, unlike most other technical activities, is required to change its state of mind (and culture, if possible) through the lifecycle of the project.

3. The interruptions are of psychological nature and should be treated as such.

4. Preparing the team for changes and for the interruptions should contribute to the smooth running of the project. 
5. Project stakeholders may participate in the management leading team of the project.

6. The project manager should be placed in an integrated position, not in a position from which he represents only a part of the problem.

Finally, according to the solutions suggested in this paper, deriving from the Gestalt COE theory, the support of the organization, the involvement of stakeholders and the capabilities of project managers in coping with interruptions are all critical to the success of the project. Since the similarities between the processes of the COE and project management were demonstrated, it is not surprising that similar findings were found in a project management research. A field study performed lately by the Standish Group, called the Chaos report (Johnson et. al., 2001), found that the three most important factors to influence project success are "executive support", "user involvement" and "an experienced project manager", as was mentioned in this paper. 


\section{Bibliography}

- Bar-Yoseph B. A. (2001). Gestalt and Engineering: A Virtual Meeting of Two Cultures. Gestalt Review, 5, 2, 88-96.

- Bar-Yoseph B. A. (2001). Culture Changes and Patterns of Management. Best Patterns - Erfolgsmuster des Zukunftsfaigen Management” (Success Patterns of Sustainable Management). Luchterhand Verlag (Publisher) Neuwied, G. \& Meurer G. (Editors) Germany.

- Clarkson P. (1989). Gestalt Counseling in Action. Sage Publishers Ltd. London, UK.

- Haris, J. (1989). Gestalt: An Idiosyncratic Introduction. Manchester Gestalt Centre.

- Houston G. (1998). The Now Red Book of GESTALT. Eights edition, Published by the author, UK.

- Johnson, J., Karen, D., Boucher, K. C. \& Robinson J. (2001). Collaboration on Project Success. Software Magazine. February-March.

- Lockyer K. \& Gordon J. (1996). Project Management and Project Network Techniques, 6th Edition, Pitman Publishing, UK.

- Maylor H. (1999). Project Management. (2 ${ }^{\text {nd }}$ Edition), Pitman Publishing, UK.

- Nevis E. C. (1987). Organizational Consulting - a Gestalt Approach. Gestalt Institute of Cleveland Press, USA.

- Nevis E. C., Lancourt J. \& Vassallo H. G. (1996). International Revolutions - a Seven Point Strategy for Transforming Organizations. Jossey Bass Inc., USA.

- PMI Standards Committee. (2000). A Guide to the Project Management Body of Knowledge. Newtown Square, PA: Project Management Institute.

- Rainey, M. A. (1993). Gestalt Resistances. The Gestalt Institute of Clevland.

- Stevenson, W. J. (2002). Operations Management. McGraw-Hill Higher Education. 
- Wheeler G. (1991). GESTALT Reconsidered. GIC press, Published by Gardner Press Inc. New York, New York, USA. 